\title{
Two-dimensional chronostratigraphic modelling of OSL ages from recent beach-ridge
} deposits, SE Australia

Tamura, Toru; Cunningham, Alastair C.; Oliver, Thomas S.N.

Published in:

Quaternary Geochronology

Link to article, DOI:

10.1016/j.quageo.2018.03.003

Publication date:

2019

Document Version

Peer reviewed version

Link back to DTU Orbit

Citation (APA):

Tamura, T., Cunningham, A. C., \& Oliver, T. S. N. (2019). Two-dimensional chronostratigraphic modelling of OSL ages from recent beach-ridge deposits, SE Australia. Quaternary Geochronology, 49, 39-44.

https://doi.org/10.1016/j.quageo.2018.03.003

\section{General rights}

Copyright and moral rights for the publications made accessible in the public portal are retained by the authors and/or other copyright owners and it is a condition of accessing publications that users recognise and abide by the legal requirements associated with these rights.

- Users may download and print one copy of any publication from the public portal for the purpose of private study or research.

- You may not further distribute the material or use it for any profit-making activity or commercial gain

- You may freely distribute the URL identifying the publication in the public portal 


\section{Accepted Manuscript}

Two-dimensional chronostratigraphic modelling of OSL ages from recent beachridges deposits, SE Australia

Toru Tamura, Alastair C. Cunningham, Thomas S.N. Oliver

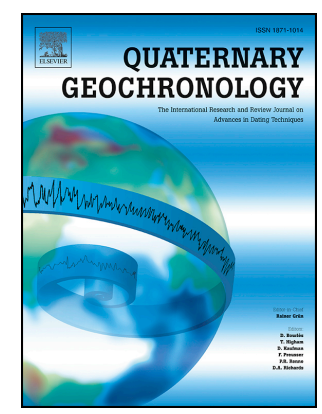

PII: $\quad$ S1871-1014(17)30239-X

DOI: $\quad$ 10.1016/j.quageo.2018.03.003

Reference: QUAGEO 901

To appear in: Quaternary Geochronology

Received Date: 6 December 2017

Revised Date: 8 March 2018

Accepted Date: 20 March 2018

Please cite this article as: Tamura, T., Cunningham, A.C., Oliver, T.S.N., Two-dimensional chronostratigraphic modelling of OSL ages from recent beach-ridges deposits, SE Australia, Quaternary Geochronology (2018), doi: 10.1016/j.quageo.2018.03.003.

This is a PDF file of an unedited manuscript that has been accepted for publication. As a service to our customers we are providing this early version of the manuscript. The manuscript will undergo copyediting, typesetting, and review of the resulting proof before it is published in its final form. Please note that during the production process errors may be discovered which could affect the content, and all legal disclaimers that apply to the journal pertain. 
1 Two-dimensional chronostratigraphic modelling of OSL ages from recent

2 beach-ridges deposits, SE Australia

4 Toru Tamura $^{\mathrm{a}, \mathrm{b}^{*}}$, Alastair C. Cunningham ${ }^{\mathrm{c}, \mathrm{d}}$, Thomas S.N. Oliver ${ }^{\mathrm{e}}$

6 a Geological Survey of Japan, National Institute of Advanced Industrial Science and

7 Technology, Central 7, 1-1-1 Higashi, Tsukuba, Ibaraki 305-8567, Japan

$8 b$ Graduate School of Frontier Sciences, The University of Tokyo, 5 Chome-1-5

9 Kashiwanoha, Kashiwa, Chiba 277-8561, Japan

10 c Nordic Laboratory for Luminescence Dating, Department of Geoscience, Aarhus

11 University, Ris $\phi$ Campus, DK-4000 Roskilde, Denmark

$12 d$ Center for Nuclear Technologies, Technical University of Denmark, Ris $\phi$ Campus,

13 DK-4000 Roskilde, Denmark

14 e School of Earth \& Environmental Science, University of Wollongong, Wollongong 15 2522, NSW, Australia

$16 *$ Corresponding author. E-mail address: toru.tamura@aist.go.jp (Toru Tamura)

\section{Abstract}

Optically-stimulated luminesecne (OSL) dating, in concert with two-dimensional ground-penetrating radar (GPR) profiling, has contributed to significant advances in our understanding of beach-ridge systems, and sedimentary landforms in various settings. For recent beach-ridges, the good OSL properties of coastal quartz permit a high sample throughput—-thanks to shorter measurement times, and simpler sample preparation-prompting the collection of more samples at higher sampling resolution. However, sampling at high resolution increases the chances of age inversions, because random errors between samples may be larger than the difference in sample ages. Age inversions can be avoided, however, if the stratigraphic constraints are included in the age estimation process. Here, we create a custom Bayesian chronological model for a recent $(<500 \mathrm{yr})$ beach-ridge sequence in Moruya, southeast Australia, for direct comparison with a GPR profile. The model includes a full 'burial-dose model' for each sample, and a dose rate term, with the modelled ages constrained by the vertical and shore-normal sample order. The modelled ages are visualized by plotting isochrones on the beach-ridge cross section, and validated against a beach monitoring dataset. The 
modelling approach allows a more detailed interpretation of the Moruya beach-ridge system; when combined with higher-resolution sampling, the approach will increase the precision of beach-ridge chronologies and provide further insights into their formative 37 processes.

38 Keywords: Baysian age model; burial dosep; beach; foredune; GPR; OSL dating 39

\section{Introduction}

OSL dating of quartz grains has proven very successful when applied to recently formed beach ridges. Constant reworking of grains on the beachface and dunes permits

43 a high degree of sunlight bleaching, and with almost no residual OSL signal, the minimum obtainable age can be as low as one or two decades (e.g., Ballarini et al., 2003). Beach ridges are also well-suited for sub-surface profiling with ground-penetrating radar (GPR). With high-relief topography, beach ridges and dunes profiled in cross section show inclined bedding surfaces, representing lateral sedimentary accretion. The combination of OSL dating and GPR profiling is especially powerful, and has enabled significant advances in our understanding of coastal or desert sedimentary landforms over the last decade (Bristow et al., 2007; Buynevich et al., 2007; Cunningham et al., 2011a; Tamura, 2012).

Nevertheless, there is currently an information mismatch between OSL and GPR data. Radar profiles provide a 2-D visualization of the bedding structures with resolution of 5-20 cm, from which the stratigraphic succession can be inferred. In contrast, OSL ages are obtained at isolated locations, perhaps on the order of 10 metres apart, and usually in one spatial dimension. In consequence, the resolution of the chronology is far lower than the resolution of the stratigraphy, meaning that important details or processes may be missed.

To resolve the discrepancy, OSL sampling could be conducted systematically in two 60 dimensions (vertically and shore-normal), with high sampling resolution; a 2D age 61 profile could then be interpolated from the 'spot' OSL ages, and overlaid on the GPR 62 image. However, OSL ages are subject to random (sample-to-sample) errors on the 63 order of $5 \%$, ensuing mostly from the dose rate measurements. At high sampling 64 resolution, the random errors in the OSL ages may be larger than the age difference 65 between samples, leading to dates that not in stratigraphic order. A 2D interpolation of 
such data will either maintain the inconsistencies, or require unfeasible numbers of samples to permit the use of a smoothing algorithm.

Age reversals can be avoided if the stratigraphic relationships between samples are used to constrain the OSL ages, such as with the commonly used Bayesian chonological tools developed for radiocarbon (e.g. Bronk-Ramsey, 2009). So far, the use of OSL data in Bayesian chronological frameworks has been restricted to the final age estimates, placed within a one-dimensional (usually age-depth) stratigraphy (Rhodes et al., 2003; Cunningham \& Wallinga, 2012; Brill et al., 2015). Here, we create a custom Bayesian chronological model for a beach-ridge sequence in Moruya, southeast Australia, which considers the measurement data in a finer detail. The model combines an aliquot-level Bayesian burial-dose model with sample-level dose-rate estimates, plus age-order constraints on the sample ages. The modelled ages are visualized by plotting isochrones on the 2-dimensional profile, validated against a dataset of beach topography changes (McLean and Shen, 2006), and used to provide further insights into the formative processes of coastal landforms.

\section{Study area and samples}

The Holocene prograded coastal plain at Moruya is located $250 \mathrm{~km}$ SSW of Sydney, SE Australia (Fig. 1A). This coastal plain is emplaced within a geologically inherited bedrock embayment and has been filled with marine sediments during the post-glacial marine transgression and subsequent regression. (Thom et al., 1981). About 60 beach ridges occur on the coastal plain, and their OSL ages reveal that the shoreline has prograded at a nearly constant rate of $0.27 \mathrm{~m} / \mathrm{yr}$ since $7200 \mathrm{yr}$ in response to the stable sea level (Oliver et al., 2015).

The coastline of the Moruya coastal plain is a 6-km long crescent-shaped beach facing ESE towards the Tasman Sea and bounded by a headland to the north and the mouth of the Moruya River to the south. It is a microtidal, moderate- to high-energy beach and composed of medium to fine sands (McLean and Shen, 2006). Since January 1972, beach profiles have been monitored along four shore-normal transects, No. 1-4 (No. 1 profile is shown in Fig. 2A; Thom and Hall, 1991; McLean and Shen, 2006). A series of extratropical cyclones in the western Tasman Sea caused extensive coastal erosion in southeastern Australia between 1974 and 1976. The Bengello Beach also experienced an extreme retreat in 1974 and following erosion-dominated period until 
1978 (Thom and Hall, 1991), and subsequently recovered in 1978-83. After 1983, the beach has exhibited a slight positive sediment budget with alternations of smaller storm erosion and subsequent recovery (McLean and Shen, 2006).

Sediment sampling for OSL dating was carried out in April 2016 along two shore-normal transects: 1) No. 1 transect of the beach monitoring, which we refer to as ANU P1 transect, and 2) Windsock transect extending $170 \mathrm{~m}$ inland from the present shoreline (Fig. 1B). Prior to the sampling, the GPR survey was undertaken along the transects with a Mala Pro Ex system equipped with a $250 \mathrm{MHz}$ antenna. Processing of GPR data was completed using RadExplorer 1.4.2 and standard processing routines were applied including desaturation, first arrival time correction, amplitude correction, bandpass filtering and topographic correction. A two-layer velocity structure was applied which accounted for changes in radar velocity associated with the groundwater table. Values of $0.14 \mathrm{~m} / \mathrm{ns}$ above and $0.07 \mathrm{~m} / \mathrm{ns}$ below the water table were adopted. Boreholes for sampling were chosen according to the GPR profiles, and dug with a steel sand auger. Sediments for OSL dating were sampled at target depths by hammering light-safe stainless tubes. 11 and 27 samples were obtained from the ANU P1 and Windsock profiles, respectively (Table S1; Figs. 2, 3).

\section{Methods}

\subsection{Sample preparation, OSL measurements and dose-rate determination}

Sample preparation and measurements for OSL dating were done at the luminescence laboratory of the Geological Survey of Japan; the details are described in the Supplementary material. Extracted quartz sand grains (180-250 $\mu \mathrm{m}$ in diameter) were moumted on a stainless disc to form a large $(8 \mathrm{~mm}$ in diameter) aliquots and measured with a TL-DA-20 automated Ris $\varnothing$ TL/OSL reader. The single-aliquot regenerative-dose (SAR) protocol was used to determine the equivalent dose $\left(D_{e}\right)$ (Murray and Wintle, 2000). Based on tests of dose recovery, dose plateau and thermal transfer, a preheat temperature of $180{ }^{\circ} \mathrm{C}$ was chosen for all samples. A cutheat was set at $160{ }^{\circ} \mathrm{C}$. The contributions of both natural radioisotopes and cosmic radiation were considered for determination of the environmental dose rate (Table S1). The final $D_{e}$ value was determined by applying the Central Age Model (Galbraith et al., 1999) for individual sample or Bayesian modelling described below, and then divided by an 
131 environmental dose rate to obtain OSL ages. All ages are expressed relative to AD 2016

132 (Table S2).

133

\subsection{Chronostratigraphic model}

135 Our aim is to build a 2-dimensional chronostratigraphic model, for which there are 136 two aspects. The first is to design a burial-dose model, which will estimate the mean 137 burial dose for a given sample from the measured $D_{\mathrm{e}}$ distribution. The second is to 138 incorporate the prior constraints provided by the stratigraphic relationship between 139 samples. As the constraints come through the age order of samples, each sample's dose 140 rate must be included as a parameter. The full model combines both of these aspects, 141 such that the age for each sample reflects the measured data (both equivalent dose and 142 dose rate), while satisfying the prior constraints. The model is built along hierachichal 143 Bayesian principals and coded in the propabilistic programming language Stan 144 (Carpenter et al., 2016).

145 OSL measurement data is typically reduced to a set of estimates of equivalent dose. 146 The measurements are subject to counting uncertainties, so are paired with an 147 uncertainty estimate. The 'measured' dose $(y)$ is an estimate of each aliquot's true 148 equivalent dose $(\theta)$, and this measurement is subject to error:

$150 y_{i}=\theta_{i}+\varepsilon_{i}$

152 for ( $\mathrm{i}=1$ to $n$ ) aliquots, where $\varepsilon$ denotes the unknown error. The likely magnitude of the 153 error is estimated by propagating the counting uncertainties, and is denoted here as $\delta_{\mathrm{i}} . \varepsilon_{\mathrm{i}}$ 154 is presumed to be drawn from a normal distribution with mean $\theta_{\mathrm{i}}$ and standard deviation $155 \delta_{\mathrm{i}}$ (see Galbraith and Roberts, 2012).

156 If all grains had received the same burial dose, and $\varepsilon$ is the only source of 157 measurement error, then the burial dose could be estimated by taking a weighted 158 average of $y$, with weights determined by $\delta$. However, it has long been recognized that 159 other sources of error influence $y$. These can be classified as intrinsic or extrinsic 160 sources (Thomsen et al., 2005). Intrinsic sources are those that arise during the 161 measurement process, creating error in excess of that expected from the counting 162 uncertainty. Extrinsic sources reflect process operating before measurement, such as 163 differential bleaching between grains, and heterogeneity in the beta dose rate in nature. 
The purpose of a dose model is to explain the measured dose distribution $(y)$ in a way that accounts for the likely sources of scatter, allowing a reasonable estimate of the parameter of interest (the mean burial dose). The model choice will depend on the dominant sources of scatter, and the measurement details. The samples considered here are quartz grains from beach and beach-ridge sediment. The grains are likely to have been very well bleached, given the speed of resetting of the quatz fast component and the opportunities for sunlight exposure during transport and deposition. Furthermore, the measurements were performed using large aliquots (>1000 grains each), so that any extrinsic variability (e.g. beta dose-rate differences between grains) will be largely removed through averaging of the signal. We therefore expect that most of the measured $y$ will be normally distributed around the mean burial dose, with the spread governed largely by the intrinsic sources of scatter. However, we can also expect some outliers in the distribution-due to e.g. occasional grains with abnormal dose-response behaviour, non-quartz grains, or occasional unbleached grains. To account for this possibility, the model includes a second normal distribution, also centred on the mean burial dose. The probability distribution is then defined as a normal mixture model:

180

$$
p(\theta)=\varphi \cdot \mathcal{N}(\mu, \sigma)+(1-\varphi) \cdot \mathcal{N}(\mu, \tau)
$$

where $N($.$) denotes the normal distribution, \mu$ is the mean burial dose (Gy), $\sigma$ is the standard deviation of the first (main) population, $\tau$ is the standard deviation of the second (outlier) population, and $\varphi$ is the proportion of $y$ corresponding to the first population. In a hierarchical Bayesian model, the unknown $\theta_{\mathrm{i}}$ are explicitly parameterized, and so the likelihood function becomes:

$$
p(y \mid \theta)=\prod_{i=1: n} \mathcal{N}\left(y_{i} \mid \theta_{i}, \delta_{i}\right)
$$

189 i.e, the unknown $\theta_{\mathrm{i}}$ are constrained by the measured $y_{\mathrm{i}}$. Computational Bayesian 190 methods evaluate the simplified Bayes equation-posterior is proportional to likelihood 191 times prior-and so we have the opportunity to specify prior knowledge to constrain the 192 parameters. Here these take three forms: those that are implicit in the model formulation, 193 those explicitly quantifying parameters, and those expressing relationships between 
194 parameters. $\varphi$ represents a proportion and so implicitly lies between 0 and 1 ; but we add

195 an explicit, informative prior of $N(1,0.1)$. The effect is to force $\varphi$ to lie close to 1 , so that

196 'outlier' population can represent only a small proportion of the data.

197 For $\sigma$, the expected spread in the main population, we can apply an informative prior 198 based on previous research. When expressed as a proportion, $\sigma$ is equivalent to the $\sigma_{\mathrm{b}}$ 199 parameter in the minimum age model (Galbraith et al., 1999). The minimum possible

200 value could be estimated with a gamma dose-recovery experiment, which usually 201 indicate a standard deviation of $\sim 8 \%$ (Thomsen et al., 2005). Additional spread must 202 come from dose-rate heterogeneity to individual grains (likely $>20 \%$ ); however, the large-aliquot averaging for our measurements must reduce this to $<10 \%$ (Cunningham et al. 2011b). Because the degree of bleaching of our samples is high, we can use the observed distribution in overdispersion of our samples to define a prior of $8.3 \pm 2.6 \%$ (supplementary figure S3). The spread in the second 'outlier' population is required to be larger, with the minimum of $20 \%$ of the burial dose. An example of the standalone Bayesian outlier model is illustrated in Fig. 4 for sample gsj16114. The burial dose is then defined as the mean and standard deviation of the posterior draws of $\mu$. For this sample, $\varphi$ is estimated as $0.88 \pm 0.06$, suggesting that some of the $y_{\mathrm{i}}$ are likely to be outliers (as is evident from the distribution).

212 For the full chronostratigraphic model, the samples are not analysed on a standalone 213 basis, but combined in a single model implementation so that between-sample 214 relationships can be included. To achieve this, a dose-rate parameter for each sample, $\beta$, 215 must be included. For each of $j$ samples, $\beta_{\mathrm{j}}$ is constrained by the measured dose rate, $z_{\mathrm{j}}$, 216 and its estimated random uncertainty $\rho_{\mathrm{j}}$. Ideally, this value should be assessed by 217 replicate measurements of a single sample. In the (not unusual) absence of such data, 218 we presume $\rho_{\mathrm{j}}$ to be $5 \%$ of the dose rate. This choice reflects our judgement that a 219 lower value would not be reasonable, given the reproducibility measurements of Murray 220 et al. (2015), and that a larger value is not necessary to explain measurement data- the 221 posterior $\beta_{\mathrm{j}}$ differ from the measured dose rates by an average of $4 \%$. The likelihood 222 term now evaluates the dose-rate parameter at the sample level, and the equivalent-dose 223 parameters at the aliquot level: 


$$
p(y, z \mid \theta, \beta)=\prod_{j=1: n}\left(\mathcal{N}\left(z_{j} \mid \beta_{j}, \rho_{j}\right) \cdot \prod_{i=1: n} \mathcal{N}\left(y_{i} \mid \theta_{i}, \delta_{i}\right)\right)
$$

227 Stratigraphic constraints are implemented as age-ordering of sample pairs, so we 228 introduce a transformed parameter $\alpha$ to represent the sample ages, where $\alpha_{\mathrm{j}}=\mu_{\mathrm{j}} / \beta_{\mathrm{j}}$. For any given $(a, b)$ pair, sample $b$ is declared older than sample $a$ through a uniform prior:

$p\left(\alpha_{b}\right)=\mathcal{U}\left(\alpha_{a}, L\right)$

232 Where $L$ is an arbitrary upper limit on the age, well outside the feasible age range for all samples. Such priors do not allow for any ambiguity in the age-relationships, so they are included only where strictly justifiable on stratigraphic grounds. Age-order pairs are indicated by arrows on the GPR profiles (Figs. 2 , 3), selected by one of three criteria:

1. Within each borehole site, age increases from top to bottom.

2. For foreshore samples of the same elevation, age increases landwards.

3. Unambiguous GPR reflectors that show the stratigraphic order.

Most age-order pairs were selected using criteria 1 and 2. Some viable age-order pairs were omitted as superfluous.

241 The parameters of interest are the sample ages $\alpha$, most easily defined by the mean and standard deviation of the posterior draws. The (marginal) posterior distribution for $\alpha_{\mathrm{i}}$ indicates the random uncertainty only; the absolute age uncertainty must include a systematic component of $\sim 5 \%$, added in quadrature.

\section{Results}

The modeled and unmodeled individual age estimates were obtained for the ANU P1 and Windsock profiles and compared with independent age evidence. For the ANU P1 profile, independent depositional age evidence is provided by the beach monitoring since AD1972 (Fig. 2C). Individual age estimates agree with the stratigraphic order both laterally and vertically (Fig. 2C), and 9 samples out of 11 samples are consistent with the independent age evidence. Two exceptions occur in the upper sample at $15 \mathrm{~m}$ and lower sample at $48 \mathrm{~m}$, which were dated $56 \pm 3 \mathrm{yr}$ and $50 \pm 4 \mathrm{yr}$, respectively; compared to the beach topography changes (Fig. 2A), these ages are overestimated by $20 \mathrm{yr}$ and $10 \mathrm{yr}$, respectively. The chronostratigraphic modelling resulted in almost 
256 identical estimates to the individual estimates. Isochrones based on modeled estimates

257 reflect the beach scarp in 1970's as well as the seaward accretion of the foredune to

258 beach before 1970 and rapid recovery after 1976 (Fig. 2D).

259 The Windsock profile largely shows individual estimates consistent with the GPR

260 stratigraphy, but contains several age reversals. These occur in the seaward accretion

261 part at an elevation c. $+2 \mathrm{~m}$ between 30-60 m, and between the middle and lower

262 samples at $40 \mathrm{~m}$. The AD 1974 scarp occurring between 80-90 m provides a measure of

263 independent age evidence. The borehole at $85 \mathrm{~m}$ penetrates the beach scarp; it is

264 probable that the upper two samples are sands deposited after the 1974 scarp while the

265 lower sample was deposited prior to 1974 . The middle sample $(51 \pm 2 \mathrm{yr}$ or AD $1965 \pm$

266 2) is thus slightly overestimated by 5-10 yr. The lower sample at $90 \mathrm{~m}(71 \pm 3 \mathrm{yr}$ or AD

267 1945 \pm 3 ) appears to occur above the pronounced scarp reflection and so should have

268 been deposited after1974. The OSL age for this sample is thus also overestimated by

$269 \sim 30$ yr. Apart from these two ages, individual age estimates are consistent with the age

270 of the beach scarp. The chronostratigraphic modelling resulted in estimates similar to

271 individual ones, but critically removed the age reversals. Isochrones after AD 1550 (Fig.

272 3C) represent the overall trend of the seaward accretion, matching well with the GPR

273 profile, and also define the building of a mound around AD 1700. Landward of the

274 mound, isochrones show the presence of the swale of which base occurs at $+2 \mathrm{~m}$ AHD

275 while the GPR reflection shows it occurs at +4.0-4.5 m AHD.

276

\section{5. Discussion}

278 The application of the Bayesian modelling removed age reversals in the Windsock 279 profile, and successfully generated an internally-consistent 2D age sequence. In contrast, 280 the modelling provided almost identical results to the unmodeled, individual estimates 281 in the ANU P1 profile, where there is no age reversal and thus the stratigraphic 282 constraint is not critical. An overestimate of the lower sample at $48 \mathrm{~m}$ of the ANU P1 283 was not modified in the modelling because of the lack of an older sample for constraint.

284 The lower sample at $90 \mathrm{~m}$ of the Windsock was also left overestimated as the adjacent 285 older sample (at $85 \mathrm{~m}$ ) was too old to give an effective constraint. These inaccuracies 286 are derived from the lack of sufficient constraint, and ao can be improved by 287 higher-resolution sampling. 
Isochrones as shown in Figs. 2D and 3C need to be defined by internally-consistent 2D age sequences, for which the chronostratigraphic modelling plays a critical role.

290 Particularly for the Windsock profile, our dataset documents the sporadic and variable

291 beach progradation and building of the isolated mound considered as the foredune,

292 providing important insights into the decadal- to centennial-scale development of the

293 landform. The most prominent inconsistency between the isochrones and GPR

294 reflection occur in the definition of the swale around $30 \mathrm{~m}$ in the Windsock profile. This

295 is caused by the interpolation between the two samples at $30 \mathrm{~m}$ of which dates show a

296 large gap, and thus additional intermediate data point should increase the reliability of 297 isochrones.

298 While higher resolution sampling will obviously be beneficial, the precision of the $2 \mathrm{D}$ age profile can also be increased by adding more constraints, and this need not relate directly to OSL sampling locations. At present, the isochrones are calculated by assuming a linear interpolation of the modelled OSL ages, using delauny triangulation.

302

303

304

305

306

307

308

309

310

311

312

313

\section{Conclusions}

We have applied two-dimensional chronostratigraphic modelling to OSL ages obtained in ground-penetrating radar profiles of beach-ridge deposits, SE Australia, and successfully generated internally-consistent age estimates. Coherent ages are critical for defining isochrones that show the decadal- to centennial-scale development of high-relief landforms in coasts and deserts. In our dataset, a few overestimated ages remained in the modelling result and the linearly interpolated isochrones were partly discordant with the GPR reflections of highly sporadic beach and foredune deposits. Taking more samples in shorter intervals, coupled with enhancements in modelling, is likely to minimise these discrepancies in future, and permit comprehensive and high-resolution chronologies. 


\section{Acknowledgements}

Prof. Colin Woodroffe is thanked for his supervision of the project. Prof. Andrew Short provided helpful suggestions as well as helped with local logistics such as accommodation. Prof. Roger McLean kindly guided us to the transects of beach monitoring at Moruya and agreed to our sampling. The project was supported by Australian Research Council Discovery Project 150101936 and by the Geological Survey of Japan, National Institute of Advanced Science and Technology. Dr Kazumi Ito and Mrs Kana Takamori are also thanked for their laboratory help.

\section{References}

Adamiec, G., Aitken,M., 1998. Dose-rate conversion factors: update. Ancient TL 16, $37-50$.

Brill, D., Jankaew, K., Brückner, H., 2015. Holocene evolution of Phra Thong's beach-ridge plain (Thailand)—chronology, processes and driving factors. Geomorphology 245, 117-134.

Bristow, C.S., Duller, G.A.T., Lancaster, N., 2007. Age and dynamics of linear dunes in the Namib desert. Geology 35, 555-558.

Bronk Ramsey, C., 2009. Bayesian analysis of radiocarbon dates. Radiocarbon 51, 337360.

Buynevich, I.V., FitzGerald, D.M., Goble, R.J., 2007. A 1500 yr record of North Atlantic storm activity based on optically dated relict beach scarps. Geology 35, 543-546.

Carpenter, B., Gelman, A., Hoffman, M., Lee, D., Goodrich, B., Betancourt, M., Brubaker, M.A., Guo, J., Li, P., Riddell, A., 2016. Stan: A probabilistic programming language. Journal of Statistical Software 20, 1-37.

Cunningham, A.C., Bakker, M.A., van Heteren, S., van der Valk, B., van der Spek, A.J., Schaart, D. R., Wallinga, J., 2011a. Extracting storm-surge data from coastal dunes for improved assessment of flood risk. Geology 39, 1063-1066.

Cunningham, A., Wallinga, J., Minderhoud, P., 2011b. Expectations of scatter in equivalent-dose distributions when using multi-grain aliquots for OSL dating. Geochronometria 38, 424-431. 
352

353

354

355

356

357

358

359

360

361

362

363

364

365

366

367

368

369

370

371

372

373

374

375

376

377

378

379

380

381

382

383

384

Galbraith, R.F., Roberts, R.G., 2012. Statistical aspects of equivalent dose and error calculation and display in OSL dating: An overview and some recommendations. Quaternary Geochronology 11, 1-27.

Galbraith, R.F., Roberts, R.G., Laslett, G.M., Yoshida, H., Olley, J.M., 1999. Optical dating of single and multiple grains of quartz from Jinmium rock shelter, northern Australia: Part I, experimental design and statistical models. Archaeometry 41, 339-364.

McLean, R.F., Shen, J., 2006. From foreshore to foredune: foredune development over the last 30 years at Moruya beach, New South Wales, Australia. Journal of Coastal Research 23, 28-36.

Murray, A.S., Wintle, A.G., 2000. Luminescence dating of quartz using an improved single-aliquot regenerative-dose protocol. Radiation Measurements 32, 57-73.

Murray, A., Buylaert, J.-P., Thiel, C., 2015. A luminescence dating intercomparison based on a Danish beach-ridge sand. Radiation Measurements 81, 32-38.

Oliver, T.S.N., Dougherty, A.J., Gliganic, L.A., Woodroffe, C.D., 2015. Towards more robust chronologies of coastal progradation: optically stimulated luminescence ages for the coastal plain at Moruya, south-eastern Australia. The Holocene 25, 536546.

Rhodes, E., Bronk Ramsey, C., Outramc, Z., Battc, C., Willisc, L., Dockrillc, S., Bond, J., 2003. Bayesian methods applied to the interpretation of multiple OSL dates: high precision sediment ages from Old Scatness Broch excavations, Shetland Isles. Quaternary Science Review 22, 1231-1244.

Tamura, T., 2012. Beach ridges and prograded beach deposits as palaeoenvironment records. Earth-Science Reviews 114, 279-297.

Thom, B.G., Hall, W., 1991. Behaviour of beach profiles during accretion and erosion dominated periods. Earth Surface Processes and Landforms 16, 113-127.

Thom, B.G., Bowman, G.M., Gillespie, R., Temple, R., Barbetti, M., 1981. Radiocarbon Dating of Holocene Beach-Ridge Sequences in South-East Australia. Department of Geography, University of New South Wales at Royal Military Colledge, Duntroon, ACT, Australia.

Thomsen, K.J., Murray, A.S., Bøtter-Jensen, L., 2005. Sources of variability in OSL dose measurements using single grains of quartz. Radiation Measurements 39, 4761. 
385

386

387

388

389 


\section{Table and Figure captions}

Fig. 1. Location of the studied profiles, Windsock and ANU P1; the inset shows the location of the satellite image.

Fig. 2. Data from the ANU P1 transect. A) Beach profiles monitored since 1972 (McLean and Shen, 2006). B) Ground-penetrating radar profile showing locations of OSL samples and direction of prior constraint. Arrows are toward the lower (and thus older) stratigraphic layer. C) Estimates of OSL ages in comparison with independent age evidence derived from the beach profile changes. The upper value indicates the individual estimate while the lower bold value shows the estimate from the modelling. D) Isochrones based on the estimates from the modelling for every decade since AD 1910. (E) Modelled ages for each sample (posterior $\alpha$ ), plotted by approximate stratigraphic location. Also plotted is a probability density function of $D_{\mathrm{e}}$, plotted on the age scale by assuming the measured dose rate (solid line); and the same when using the modelled dose rates (dashed line). The unmodelled ages (CAM / measured dose rate) are indicated in red.

Fig. 3. Data from the Windsock transect. A) Ground-penetrating radar profile showing locations of OSL samples and direction of prior constraint. Arrows are toward the lower (and thus older) stratigraphic layer. B) OSL ages based on the individual estimate (the upper value) and modeled estimate (the lower bolded value). C) Isochrones based on the estimates from the modelling for every $50 \mathrm{yr}$ since $\mathrm{AD}$ 1550 .

Fig. 4. A) Illustration of the standalone burial dose model applied to sample gsj16114. The $D_{\mathrm{e}}$ distribution is illustrated using probability density function. The posterior $\mu$ provides the burial dose estimate (defined by its mean and standard deviation). B) Also illustrated (schematically) are the relative densities of the inferred normal populations $(\varphi=0.88)$. 
421 Supplementary Table S1. Optically stimulated luminescence (OSL) samples and data

422 for dose rate estimates: the distance in transect, depth, contents of radionuclides, $423 \quad$ water content and cosmic dose rate.

424

425 Supplementary Table S2. OSL dating results of unmodelled, individual estimates and 426 modelled estimate: dose rate, equivalent dose $\left(D_{e}\right)$ and OSL age.

427

428 Supplementary Figure S3: Histogram of overdispersion values of all samples (ANU and 429 Windsock profiles). The cluster of 33 samples with similar overdispersion values 430 are presumed to indicate the likely spread in the absence of significant outliers. 431 Their mean and standard deviation $(0.083,0.026)$ is used to specify an informative 432 prior for $\sigma$ in the Bayesian burial dose model. 


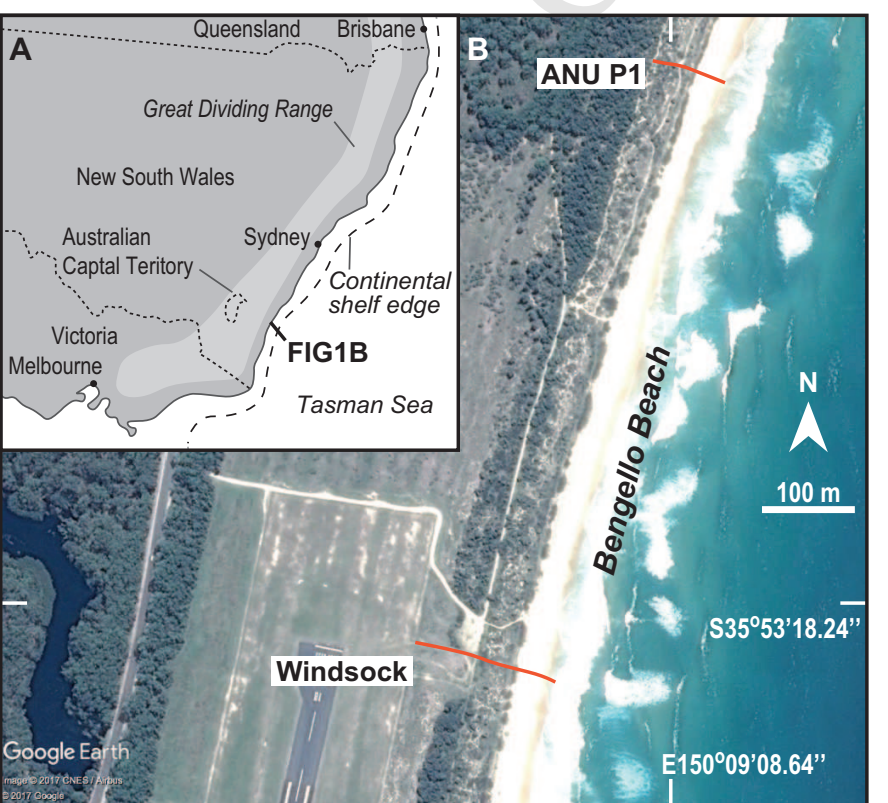


A 

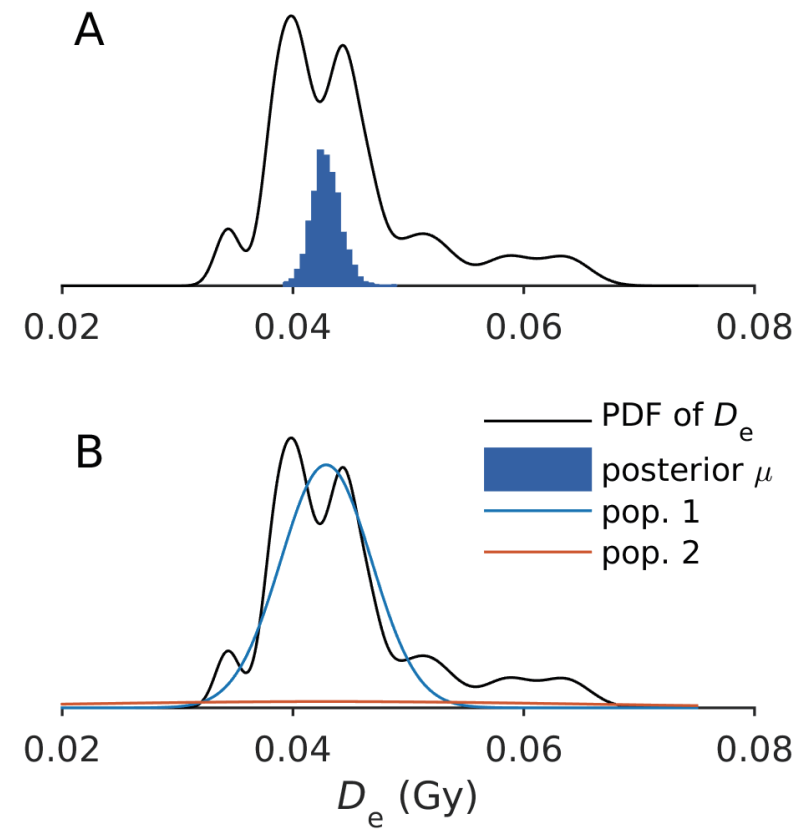